\title{
$\begin{array}{ll}\text { Research Square } & \begin{array}{l}\text { Preprints are preliminary reports that have not undergone peer review. } \\ \text { They should not be considered conclusive, used to inform clinical practice, } \\ \text { or referenced by the media as validated information. }\end{array}\end{array}$
}

\section{A Comparison Between Tau and Amyloid- $\beta$ Cerebrospinal Fluid Biomarkers in Chronic Traumatic Encephalopathy and Alzheimer Disease}

\author{
Katherine W. Turk \\ VA Boston Health Care System Jamaica Plain Campus https://orcid.org/0000-0002-6833-5494

\section{Alexandra Geada} \\ Boston University School of Medicine
}

\section{Victor E. Alvarez}

Boston University Alzheimer's Disease Center

\section{Weiming Xia}

Boston University Alzheimer's Disease Center

Jonathan D. Cherry

Boston University Alzheimer's Disease Center

\section{Raymond Nicks}

Boston University Alzheimer's Disease Center

\section{Gaoyuan Meng}

VA Boston Healthcare System

\section{Sarah Daley}

Boston University Alzheimer's Disease Center

Yorghos Tripodis

Boston University Alzheimer's Disease Center

\section{Bertrand R. Huber}

Boston University Alzheimer's Disease Center

\section{Andrew E. Budson}

Boston University Alzheimer's Disease Center

\section{Brigid Dwyer}

Boston University Medical Center: Boston Medical Center

\section{Neil W. Kowall}

Boston University Alzheimer's Disease Center

\section{Robert C. Cantu}

Boston University Alzheimer's Disease Center

\section{Lee E. Goldstein}

Boston University Alzheimer's Disease Center

Douglas I. Katz

Boston University School of Medicine

Robert A. Stern

Boston University Alzheimer's Disease Center

Michael L. Alosco

Boston University Alzheimer's Disease Center 


\section{Jesse Mez}

Boston University Alzheimer's Disease Center

\section{Ann C. McKee}

Boston University Alzheimer's Disease Center

\section{Thor D. Stein ( $\nabla$ tdstein@bu.edu )}

Boston University Alzheimer's Disease Center https://orcid.org/0000-0001-6954-4477

\section{Research}

Keywords: cerebrospinal fluid, biomarkers, Chronic Traumatic Encephalopathy, Alzheimer's disease, amyloid beta, tau

Posted Date: May 18th, 2021

DOI: https://doi.org/10.21203/rs.3.rs-521914/v1

License: @ (i) This work is licensed under a Creative Commons Attribution 4.0 International License. Read Full License

Version of Record: A version of this preprint was published at Alzheimer's \& Dementia on December 1st, 2021. See the published version at https://doi.org/10.1002/alz.051392. 


\section{Abstract \\ Background}

Cerebrospinal fluid (CSF) tau and beta-amyloid levels in chronic traumatic encephalopathy (CTE), a disease which can be clinically indistinguishable from Alzheimer's disease (AD), are largely unknown. We examined postmortem CSF analytes among participants with autopsy confirmed CTE and AD.

\section{Methods}

In this cross-sectional study 192 participants from the Boston University AD Research Center, VA-BU-CLF Center, and Framingham Heart Study (FHS) had post-mortem CSF collected at autopsy. Participants were divided into pathological groups based on AD and CTE criteria, with 61 CTE participants (18 low, 43 high stage), 79 AD participants (23 low, 56 intermediate to high), 11 participants with CTE combined with $A D$, and 41 participants lacking both CTE and AD neuropathology. The Meso Scale Discovery immunoassay system was utilized to measure amyloid-beta $\left(A \beta_{1-40}, A \beta_{1-}\right.$ $42)$, total tau (t-tau), and phosphorylated tau ( $\mathrm{p}-\operatorname{tau}_{181}$ and $\left.\mathrm{p}-\mathrm{tau}_{231}\right)$. CSF analytes were then compared across the six pathological groups: no CTE/no AD, Low CTE, Low AD, High CTE, Intermediate/High AD, and AD + CTE.

\section{Results}

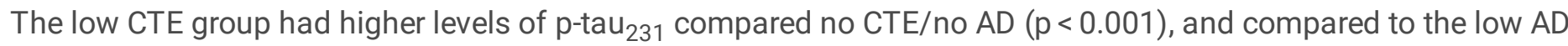
group ( $p=0.002)$. The low CTE group had lower levels of $A \beta_{1-42}$ compared to no CTE/no $A D(p=0.009)$. The high CTE group had higher levels of $p$-tau 231 compared to intermediate/high AD $(p<0.001)$.

\section{Conclusions}

Importantly, p-tau 231 and $A \beta_{1-42}$ were predictors of diagnosis of CTE vs. no CTE/no AD and CTE vs. AD. Increased CSF p-tau ${ }_{231}$ is a promising potentially sensitive biomarker of CTE, and CSF $A \beta_{1-42}$ needs further investigation in CTE.

\section{Background:}

Chronic Traumatic Encephalopathy (CTE) and Alzheimer disease (AD) are neurodegenerative conditions causing memory loss that can only be definitively diagnosed by pathologic examination. While both disease processes involve considerable tau protein deposition, amyloid- $\beta$ plaque formation is considered a prominent feature only in AD. Total tau (t-tau), phosphorylated tau (p-tau), and beta-amyloid $(A \beta)$ measurements in cerebrospinal fluid (CSF) have proven to be reliable biomarkers of $A D$ and reflect changes in brain pathology that precede cognitive decline ante-mortem (14). Biomarkers with the ability to detect and distinguish CTE from AD would be of great value if they show a high degree of correlation with CTE neuropathology.

We hypothesized that CTE participants may have increased t-tau, $\mathrm{p}$-tau ${ }_{181}$ and $\mathrm{p}$-tau $\mathrm{tan}_{23}$ compared to cases without either CTE or $A D$ (no CTE/no AD), as well as relatively higher $p$-tau ${ }_{181}, p$-tau $\tan _{231}$ and $A \beta_{42}$ concentrations compared to $A D$ cases. Prior studies of $A \beta$ deposition in CTE (5) led us to hypothesize that CSF $A \beta_{1-40}$ would be decreased in cases with co-occurring CTE and AD compared to non-AD/ non-CTE cases, representing a potential interaction between $A D$ and CTE. To test these hypotheses, we measured CSF levels of $A \beta_{1-40}, A \beta_{42}$, $t$-tau, $p$-tau 181 and $p$-tau 231 
among autopsy-confirmed participants including 61 with CTE, 79 with AD, 11 with concurrent CTE and AD, and 41 control participants lacking both CTE and AD pathology. Because the underlying neuropathology is markedly different in early and late disease, we compared CTE to AD CSF analytes separately in early and late stage disease.

\section{Methods:}

Participants

192 participants with post-mortem CSF available were enrolled from three study groups. 100 donated their brains to the Veteran's Affairs-Boston University-Concussion Legacy Foundation Brain Bank (VA-BU-CLF) as part of the Understanding Neurologic Injury and Traumatic Encephalopathy (UNITE) study, 38 were donated to the Framingham Heart Study (FHS), and 54 were donated to Boston University's Alzheimer's Disease Research Center (ADRC) as part of the Health Outreach Program for the Elderly study. The UNITE group consisted of participants with a history of exposure to contact sports such as football, ice hockey, boxing, soccer, rugby, and martial arts at either the professional or amateur level (6). For most brain donations, the next of kin contacted the brain bank to donate tissue at or near the time of death. The participants from Boston University's Alzheimer's Disease Research Center (BU ADRC) with and without cognitive impairment underwent annual cognitive evaluations using the National Alzheimer's Disease Coordinating Center (NACC) Uniform Data Set (UDS) protocol (7). The third cohort consisted of participants from the Framingham Heart Study (FHS), a longitudinal, community-based study. Consents for brain donation and research participation were provided by donor next of kin.

Head Injury Exposure Assessment

For UNITE study participants, retrospective clinical evaluations were performed using semi-structured post-mortem interviews, through online surveys, and review of medical records as described previously (6). Information was obtained regarding repetitive head impact $(\mathrm{RHI})$ exposure, traumatic brain injury (TBI) exposure, military history, athletic history, and clinical symptoms prior to death. In addition, medical records were examined to provide a determination of clinical symptoms and course. For the FHS participants, an athletic history assessment identical to UNITE was performed with the donor's next of kin(8). Athletic history was not available for BU ADRC participants. All interviews were conducted independently and blinded to the results of neuropathological examination.

Pathological criteria

All brains were neuropathologically evaluated for changes consistent with $C T E, A D$, and other neurodegenerative disorders using previously described selection criteria and protocols. Specifically, participants were separated into pathologic groups: CTE, AD, both (CTE + AD), or neither (no CTE/no AD). Pathologic diagnosis of CTE was based on consensus criteria(7) and CTE staging IIV was determined using previously published staging criteria $(9,10)$. Participants were stratified using NIA-Reagan criteria to high, intermediate, or low probability of dementia caused by $A D$, based on Braak Score and CERAD score.

There is growing recognition that with increasing age co-occurring neurodegenerative pathologies become more common $(11,12)$, therefore comorbid neurodegenerative pathologies were not excluded from any of the groups, including the no CTE/no AD group. Other neurodegenerative diseases were diagnosed using well-established criteria for Lewy body disease (LBD)(13), and frontotemporal lobar degeneration (FTLD)(14, 15) (Table 1). Participants with Amyotrophic Lateral Sclerosis/ Motor neuron disease (ALS/MND) pathology were also not excluded and included two participants in the no CTE/no AD group and one participant in the High CTE group. The inclusion of a wider group of participants with concurrent neurodegenerative pathologies increases the generalizability of the current findings as

Page $4 / 21$ 
patients presenting for clinical evaluation of cognitive complaints often have multiplane neurodegenerative diagnoses and underlying pathologies. 
Table 1

Demographic and exposure characteristics of participant groups.

\begin{tabular}{|c|c|c|c|c|c|c|c|}
\hline & $\begin{array}{l}\text { No } \\
\text { CTE/No } \\
\text { AD }\end{array}$ & Low CTE & High CTE & Low AD & $\begin{array}{l}\text { Int/High } \\
A D\end{array}$ & $\begin{array}{l}\text { CTE + } \\
\text { AD }\end{array}$ & $p$ \\
\hline Sample size (n) & 41 & 18 & 43 & 23 & 56 & 11 & \\
\hline $\begin{array}{l}\text { Age at death } \\
\text { (S.E.M.) }\end{array}$ & $\begin{array}{l}74.1 \\
(3.0)^{d}\end{array}$ & \multirow{2}{*}{$\begin{array}{l}64.6(4.6)^{f, d, e} \\
89-25\end{array}$} & \multirow{2}{*}{$\begin{array}{l}74.8(1.3)^{d} \\
90-53\end{array}$} & \multirow{2}{*}{$\begin{array}{l}86.7(1.1)^{a, b, c} \\
95-72\end{array}$} & $\begin{array}{l}81.1 \\
(1.3)^{\mathrm{b}}\end{array}$ & $\begin{array}{l}79.6 \\
(2.8)^{\mathrm{b}}\end{array}$ & \multirow[t]{2}{*}{$<.001$} \\
\hline $\begin{array}{l}\text { Age Range (Max- } \\
\text { Min) }\end{array}$ & $101-17$ & & & & $98-54$ & $70-100$ & \\
\hline \multicolumn{8}{|l|}{ Cohort: } \\
\hline FHS & 13 & 1 & 0 & 10 & 13 & 1 & \\
\hline UNITE & 17 & 17 & 43 & 1 & 12 & 10 & \\
\hline HOPE & 11 & 0 & 0 & 12 & 31 & 0 & \\
\hline Sex m/f & $33 / 8$ & $18 / 0$ & $43 / 0$ & $14 / 9$ & $27 / 29$ & $10 / 1$ & $<.001$ \\
\hline (\%male) & $(80.5 \%)^{\mathrm{c}, \mathrm{e}}$ & $(100 \%)^{e}$ & $(100 \%)^{a, d, e}$ & $(60.9 \%)^{c}$ & $\begin{array}{l}(48.2 \%) \\
a, b, c\end{array}$ & $(90.9 \%)$ & \\
\hline \multicolumn{8}{|l|}{ CTE Stage } \\
\hline Stage I & 0 & $6(33.3 \%)$ & 0 & 0 & 0 & \multirow{2}{*}{$\begin{array}{l}1 \\
(9.1 \%)\end{array}$} & \\
\hline Stage II & 0 & $12(66.7 \%)$ & 0 & 0 & 0 & & \\
\hline Stage III & 0 & 0 & $18(41.9 \%)$ & 0 & 0 & $\begin{array}{l}1 \\
(9.1 \%)\end{array}$ & \\
\hline \multirow[t]{2}{*}{ Stage IV } & 0 & 0 & $25(58.1 \%)$ & 0 & 0 & 0 & \\
\hline & & & & & & $\begin{array}{l}9 \\
(81.8 \%)\end{array}$ & \\
\hline \multicolumn{8}{|l|}{ Braak Score } \\
\hline 0 & \multirow{2}{*}{$\begin{array}{l}15 \\
(36.6 \%)\end{array}$} & 7 (38.9\%) & $1(2.3 \%)$ & 0 & 0 & 0 & \\
\hline$H$ & & $5(27.8 \%)$ & $4(9.3 \%)$ & $6(26.1 \%)$ & 0 & 0 & \\
\hline III-IV & $\begin{array}{l}12 \\
(29.3 \%)\end{array}$ & $5(27.8 \%)$ & 31 (72.1\%) & $17(73.9 \%)$ & $\begin{array}{l}7 \\
(12.5 \%)\end{array}$ & $\begin{array}{l}1 \\
(9.1 \%)\end{array}$ & \\
\hline V-VI & $\begin{array}{l}14 \\
(34.1 \%) \\
0\end{array}$ & $1(5.6 \%)$ & $7(16.3 \%)$ & 0 & $\begin{array}{l}49 \\
(87.5 \%)\end{array}$ & $\begin{array}{l}10 \\
(90.9 \%)\end{array}$ & \\
\hline \multicolumn{8}{|l|}{ CERAD Score } \\
\hline 0 & $\begin{array}{l}41 \\
(100 \%)\end{array}$ & $14(77.8 \%)$ & $21(48.8 \%)$ & 0 & 0 & 0 & \\
\hline 1 & 0 & $4(22.2 \%)$ & $22(51.2 \%)$ & $22(95.7 \%)$ & $\begin{array}{l}8 \\
(14.3 \%)\end{array}$ & $\begin{array}{l}1 \\
(9.1 \%)\end{array}$ & \\
\hline 2 & 0 & 0 & 0 & $1(4.3 \%)$ & 26 & & \\
\hline 3 & 0 & 0 & 0 & 0 & $\begin{array}{l}(46.4 \%) \\
22 \\
(39.3 \%)\end{array}$ & $\begin{array}{l}(54.5 \%) \\
4 \\
(36.4 \%)\end{array}$ & \\
\hline
\end{tabular}




\begin{tabular}{|c|c|c|c|c|c|c|c|}
\hline & $\begin{array}{l}\text { No } \\
\text { CTE/No } \\
\text { AD }\end{array}$ & Low CTE & High CTE & Low AD & $\begin{array}{l}\text { Int/High } \\
A D\end{array}$ & $\begin{array}{l}\text { CTE + } \\
\text { AD }\end{array}$ & $p$ \\
\hline $\begin{array}{l}\text { FTLD Pathology } \\
\text { Tau } \\
\text { TDP } 43 \\
\text { n total }\end{array}$ & $\begin{array}{l}10 \\
(27.7 \%)^{d, e} \\
9(25 \%) \\
1(2.7 \%) \\
36\end{array}$ & $\begin{array}{l}5(27.8 \%)^{d, e} \\
5(27.8 \%) \\
5(27.8 \%) \\
18\end{array}$ & $\begin{array}{l}6(14.6 \%) \\
6(14.6 \%) \\
4(9.8 \%) \\
41\end{array}$ & $\begin{array}{l}0(0 \%)^{a, b} \\
0 \\
0 \\
15\end{array}$ & $\begin{array}{l}3 \\
(5.4 \%)^{a, b} \\
2(4.3 \%) \\
2(4.3 \%) \\
46\end{array}$ & $\begin{array}{l}2 \\
(18.2 \%) \\
2 \\
(18.2 \%) \\
1(9 \%) \\
11\end{array}$ & $<.05$ \\
\hline $\begin{array}{l}\text { LBD Pathology } \\
\text { Brainstem } \\
\text { Limbic/Neocortical } \\
\text { n }\end{array}$ & $\begin{array}{l}2(4.4 \%) \\
4(9.8 \%) \\
36\end{array}$ & $\begin{array}{l}1(5.6 \%) \\
2(11.1 \%) \\
18\end{array}$ & $\begin{array}{l}6(14 \%) \\
10(23.3 \%) \\
43\end{array}$ & $\begin{array}{l}2(13.3 \%) \\
1(6.6 \%) \\
15\end{array}$ & $\begin{array}{l}2 \\
(4.34 \%) \\
8 \\
(17.4 \%) \\
46\end{array}$ & $\begin{array}{l}0(0 \%) \\
4 \\
(36.4 \%) \\
11\end{array}$ & 0.31 \\
\hline $\begin{array}{l}\text { Contact sports } \\
\text { play }\end{array}$ & $\begin{array}{l}\text { Yes 12 } \\
(29.2 \%) \\
\\
\text { No 2 } \\
(4.8 \%) \\
\text { Missing } \\
27 \\
(70.7 \%)\end{array}$ & $\begin{array}{l}\text { Yes } 17 \\
(94.4 \%) \\
\text { No } 0 \\
\text { Missing } 1 \\
(5.6 \%)\end{array}$ & $\begin{array}{l}\text { Yes } 43(100 \%) \\
\text { No } 0 \\
\text { Missing } 0\end{array}$ & $\begin{array}{l}\text { Yes } 0 \\
\text { No } 1(4 \%) \\
\text { Missing } 22 \\
(96 \%)\end{array}$ & $\begin{array}{l}\text { Yes } 9 \\
(16 \%) \\
\\
\text { No } 1 \\
(1.7 \%) \\
\text { Missing } \\
46 \\
(82 \%)\end{array}$ & $\begin{array}{l}\text { Yes } 10 \\
(90 \%) \\
\text { No NA } \\
\text { Missing } \\
1 \\
(9.1 \%)\end{array}$ & .013 \\
\hline $\begin{array}{l}\text { CSF Hemoglobin } \\
\text { (S.E.M.) } \\
\text { n }\end{array}$ & $\begin{array}{l}481.0 \\
(106.6) \\
36\end{array}$ & $\begin{array}{l}648.3 \\
(261.2) \\
11\end{array}$ & $\begin{array}{l}465.1 \\
(106.5) \\
33\end{array}$ & $\begin{array}{l}555.6 \\
(183.6) \\
23\end{array}$ & $\begin{array}{l}386.5 \\
(93.1) \\
49\end{array}$ & $\begin{array}{l}370.5 \\
(192.7) \\
11\end{array}$ & 0.85 \\
\hline $\begin{array}{l}\text { Postmortem } \\
\text { Interval (h:m) } \\
\text { (S.E.M) } 95 \% \mathrm{Cl} \\
\text { N }\end{array}$ & $\begin{array}{l}22: 40 \\
(3: 07)^{b, c} \\
16: 19- \\
29: 02\end{array}$ & $\begin{array}{l}46: 31 \\
(6: 43)^{a, d, e} \\
32: 15- \\
60: 46\end{array}$ & $\begin{array}{l}37: 35(2: 32)^{\text {ad,e }} \\
32: 26-42: 44 \\
36\end{array}$ & $\begin{array}{l}21: 24 \\
(3: 50)^{b, c} \\
13: 25- \\
29: 22\end{array}$ & $\begin{array}{l}19: 59 \\
(2: 19)^{\mathrm{b}, \mathrm{c}} \\
(15: 20- \\
24: 39)\end{array}$ & $\begin{array}{l}29: 21 \\
(4.01) \\
20: 22- \\
38: 19\end{array}$ & $<.001$ \\
\hline $\begin{array}{l}\text { PMI Range } \\
\text { (Max-Min) }\end{array}$ & $\begin{array}{l}35 \\
77: 00- \\
1: 45\end{array}$ & $\begin{array}{l}17 \\
101-5: 35\end{array}$ & $71: 00-5: 00$ & $\begin{array}{l}23 \\
96: 00-5: 40\end{array}$ & $\begin{array}{l}53 \\
99: 10- \\
1: 45\end{array}$ & $\begin{array}{l}11 \\
48: 00- \\
3: 00\end{array}$ & \\
\hline $\begin{array}{l}\text { RIN (S.E.M.) } \\
\text { n }\end{array}$ & $\begin{array}{l}6.2(0.2) \\
35\end{array}$ & $\begin{array}{l}5.76(0.5) \\
16\end{array}$ & $\begin{array}{l}5.6(0.2) \\
31\end{array}$ & $\begin{array}{l}5.7(0.5) \\
12\end{array}$ & $\begin{array}{l}5.8(0.2) \\
39\end{array}$ & $\begin{array}{l}4.7 \\
(0.4) \\
10\end{array}$ & 0.15 \\
\hline $\begin{array}{l}\text { pH (S.E.M.) } \\
\mathbf{n}\end{array}$ & $\begin{array}{l}6.14 \\
(0.08) \\
26\end{array}$ & $\begin{array}{l}6.14(0.10) \\
14\end{array}$ & $\begin{array}{l}6.17(0.1) \\
24\end{array}$ & $\begin{array}{l}6.07(0.13) \\
11\end{array}$ & $\begin{array}{l}6.08 \\
(0.06) \\
36\end{array}$ & $\begin{array}{l}5.97 \\
(0.8) \\
6\end{array}$ & 0.83 \\
\hline
\end{tabular}




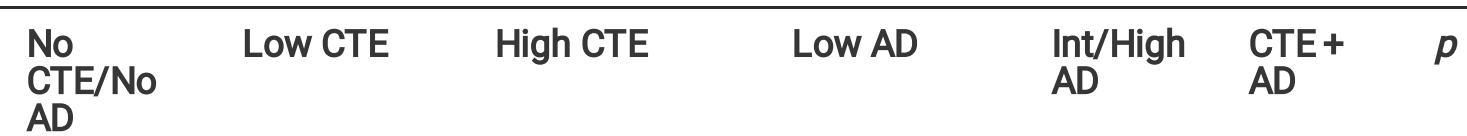

Data are presented mean with standard error of the (S.E.M.), years for age at death and contact sports exposure and as \# yes/\# no (\%) unless otherwise indicated. CERAD plaque density was rated as none (0), sparse (1), moderate (2), or frequent (3) for neuritic plaques. Int/High: Intermediate/High, AD: Alzheimer disease; CERAD: Consortium to Establish a Registry for Alzheimer's disease; CTE: chronic traumatic encephalopathy; FTLD:

Frontotemporal lobar degeneration; LBD: Lewy body disease; RIN: RNA integrity number; \#ANOVA with Bonferroni correction, ${ }^{\star} \chi 2$ test for proportions between all pathology groups.

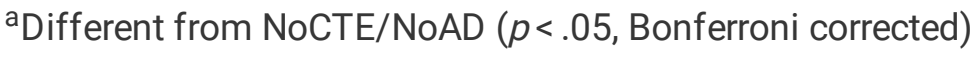

${ }^{\mathrm{b}}$ Different from Low CTE $(p<.05$, Bonferroni corrected)

'Different from High CTE ( $p<.05$, Bonferroni corrected)

${ }^{\mathrm{d}}$ Different from Low AD $(p<.05$, Bonferroni corrected $)$

eDifferent from $\mathrm{AD}(p<.05$, Bonferroni corrected $)$

${ }^{f}$ Different from CTE $+A D(p<.05$, Bonferroni corrected $)$

Participants were divided into pathological groups based on NIA-Reagan Criteria and CTE stage as follows.

Participants with no evidence of CTE and no elements of NIA-Reagan were labeled as "No CTE/No AD". Participants with CTE Stage of I-II were determined to have early stage disease and termed "Low CTE", while those with CTE Stage of III-IV were determined to have late stage disease and were termed "High CTE". Those with no evidence of CTE and NIA-Reagan of high or intermediate probability were termed "Intermediate/High AD", while those with no evidence of CTE and NIA-Reagan of low probability were classified as "Low AD". Subjects with CTE and intermediate or high probability of $A D$ were combined to "CTE + AD".

CSF sampling and analysis

CSF was obtained post-mortem from the foramen magnum by gently lifting the frontal lobes to access with a large bore needle. CSF was then mixed by gently inverting the tube 5 times. The tubes were centrifuged at $1,500 \mathrm{~g}$ for 15 minutes at $4^{\circ} \mathrm{C}$. The CSF supernatant was removed with a transfer pipet and aliquoted into $1.5 \mathrm{~mL}$ microcentrifuge polypropylene tubes. CSF was stored at $-80 \mathrm{C}$ prior to use. CSF was then diluted 1:2 with 1\% Blocker A (MSD, Rockville, MD, USA, \#R93BA) in wash buffer. Immunoassay was performed for $A \beta_{1-42}$ and $A \beta_{1-40}$, using a multiplex plate from MSD (\#K15200E), as well as for levels of p-tau 231 and total tau (MSD \#K15121D) according to manufacturer's protocol. To capture tau phosphorylated at Thr residue 181, antibody AT270 was used and the detecting antibody was the biotinylated HT7 that recognizes residue 159-163 of tau (Thermo Scientific, Rockford, IL). For hemoglobin quantification, CSF was diluted 1:3000 and applied to the RayBio Human Hemoglobin ELISA kit (\# ELH-Hgb). All standards and samples were run in duplicate. Values were standardized against the mean no CTE / no AD group to generate relative fold change.

Tissue sampling and analysis

The buffer conditions, protease inhibitors and centrifugation protocols have been reported previously (5). A 4mm tissue punch was used to isolate and remove gray matter from the gyral crests and sulcal depths of the middle frontal gyrus and neighboring sulci and superior temporal gyrus and sulcus. Brain tissue was homogenized in five-fold 
volume of $5 \mathrm{M}$ Guanidine Hydrocholride/50 mM Tris-HCL, pH 8.0, with protease inhibitors (Thermo Scientific, 78439) and phosphatase inhibitors (Sigma, P5726 and P0044). Tissue was homogenized using a mechanical homogenizer for 25 strokes followed by ultrasonic disruption on ice. The homogenates were shaken at room temperature overnight. The lysate was diluted 1:80 with 1\% Blocker A (MSD, \#R93BA) in wash buffer, and immunoassay was performed for $A \beta_{1-42}$ using a multiplex plate from MSD.

\section{Statistical analysis}

Statistical analysis was performed using SPSS 26.0 (IBM Corp, Armonk, NY) and Prism v8 (Graph-Pad Software, La Jolla, CA). A one-way analysis of variance (ANOVA) was used to compare age among groups. Relative amounts of $A \beta_{1-40}, A \beta_{1-42}, p-t_{10 u}, p-$ tau $_{231}$, and total tau were determined by dividing each measurement by the mean of the no CTE/no $A D$ group. Values that were outside $3 X$ the interquartile range were eliminated as outliers and included: $n=2$ from no CTE/no AD group, $n=1$ from Low CTE group, $n=1$ High CTE group, $n=1$ from Low AD group, and $n=1$ from Intermediate/High $A D$ group for ptau 181 analysis, $n=2$ from no CTE/no $A D$ group, $n=1$ from Low $A D$ group, $n=5$ from Intermediate/High $A D$ group, and $n=1$ from $C T E+A D$ group for ptau 231 analysis, $n=2$ from no $C T E / n o ~ A D$ group, $n=2$ from High CTE group, $n=1$ from CTE + AD group for total tau analysis, $n=5$ from no CTE/no AD group, $n$ $=3$ from Low CTE group, $n=5$ from High CTE group, $n=1$ from Intermediate/High AD group for $A \beta_{1-42}$ analysis, $n=2$ from no CTE/no $A D$ group, $n=1$ from Low CTE group, $n=3$ from High CTE group, $n=1$ from Low $A D$ group, and $n=2$ from Intermediate/High $A D$ group for $A \beta_{1-40}$ analysis. A two-sample Chi square test weighted by sample size was used to compare the frequency of men in each pathologic group, as well as the frequency of FTLD and LBD pathologies between pathologic groups. One-way ANCOVAs were performed correcting for age as a covariate to compare relative amount of biomarkers between groups. Statistical significance was set to $p<0.05$ for all planned analyses except for pairwise post-hoc comparisons following ANCOVAs determined where cutoff was Bonferonniadjusted to $p<0.003$ for five group comparisons, and $p<0.016$ for sub-group comparisons. Binary logistic regression analyses were used to determine association between $p$-tau 231 and $A \beta_{1-42}$ and CTE or $A D$ pathologic diagnosis controlling for age, sex, $\mathrm{PMI}$ and other variables where appropriate. Linear regressions were performed to determine the relationship between CSF and brain $A \beta_{1-42}$ levels in AD and CTE. Receiver operating characteristic (ROC) curve analysis was used to determine sensitivity and specificity of CSF analytes between diagnoses.

\section{Results:}

\section{Study population}

Participants were grouped based on the presence or absence of CTE and/or AD pathology. Group demographic differences for age at death, post-mortem interval (PMI), sex, RNA integrity number (RIN), pH and presence/absence of FTLD and LBD pathologies are listed in Table 1. Pathologic groups differed in age at death $(p<0.001)$ and PMI $(p<$ 0.001) (Table 1). The Low CTE group ( $M=64.6 \pm 4.6)$ was younger than the Low $A D(M=86.7 \pm 1.1, p<.05)$, Intermediate/High $A D(M=81.1 \pm 1.3, p<0.05)$, and $C T E+A D$ groups $(M=79.6 \pm 2.8, p<0.05)$. The Low $A D$ group was older than the High CTE and no CTE/no AD groups. Both the Low and High CTE groups had significantly longer PMIs $(M=46: 31 \pm 6: 43 ; M=37: 35 \pm 2: 32$ respectively) than the no $C T E /$ no $A D(M=22: 40 \pm 3: 07, p<.05)$, Low $A D(M=$ 21:24 $\pm 3: 50, p<0.05)$, and Intermediate/High AD groups $(M=19: 59 \pm 2: 19, p<0.05)$. Despite these differences in PMI, there was no difference in RIN, pH, or CSF hemoglobin between groups. A majority of men were present in each pathological group for all groups except for the Intermediate/High AD group which had a majority of women. Years of contact sports differed between groups $(p=0.013)$ for the subset of participants that had this clinical history taken (n 
= 95); post-hoc pairwise comparisons revealed the no CTE/no AD group ( $M=9.46 \pm 2.97)$ had decreased years of contact sports compared to mild CTE group $(M=20.85 \pm 3.43, p=0.036$ Bonferroni-corrected $)$.

CSF

\section{ALL GROUPS}

Comparing all pathological groups using a one-way ANCOVA correcting for age, there were significant differences in p$\operatorname{tau}_{231} F(5,177)=6.404, p<0.001$ and $A \beta_{1-42} F(5,153)=3.27, p=0.005$. The Low CTE group had significantly higher amount of $\mathrm{p}-\mathrm{tau}_{231}$ compared to both the Intermediate/High AD $(\mathrm{p}<0.001)$ and the Low AD groups $(\mathrm{p}<.001)$.

Furthermore, the Low CTE group was found to have increased levels of $p$-tau t31 compared to the High CTE group $(p=$ 0.002) as well as the CTE + AD ( $p=.002)$ and no CTE/no AD groups $(p<0.001)$ (Supplemental Table e-1).

The High CTE group was found to have significantly lower levels of $A \beta_{1-42}$ compared to no CTE/no $A D(p<0.003)$. In the Low CTE group there was a trend towards a decrease in $A \beta_{1-42}$ compared to the no CTE/no AD group $(p<0.008$, where cutoff for multiple comparisons was $p<0.003)$. As expected, $A \beta_{1-42}$ was lower in both Low and Intermediate/High AD compared to no AD/no CTE. There was no significant difference in relative amounts of $p$-tau ${ }_{181}$, total tau, and $A \beta_{1-40}$ between all groups (Supplemental Table e-1). We also performed additional ANCOVAs including age, PMI and sex as covariates to better account for group differences and potential confounders and found similar results (See supplemental results).

\section{EARLY STAGE DISEASE}

Comparing no CTE/no AD, Low CTE, and Low AD pathological groups correcting for age, differences in p-tau 231 $F(2,74)=7.84, p<0.001$ and $A \beta_{1-42} F(2,64)=4.99, p=0.01$ were found. The Low CTE group had significantly higher levels of $p-\operatorname{tau}_{231}$ versus the no CTE/no AD group $(p<0.001)$, and compared to the Low AD group $(p=0.002)(F i g .1)$. The Low CTE group was also found to have significantly lower levels of $A \beta_{1-42}$ compared to the no CTE/no $A D$ group $(p=0.009)$ (Fig. 1). The Low AD group had lower levels of $A \beta_{1-42}$ compared to the no CTE/no $A D$ group $(p=0.035)$, but this difference was not significant when corrected for multiple comparisons where the cut off was $p<0.016$. There was no significant difference in relative amount of $p-\operatorname{tau}_{181}$, total tau, or $A \beta_{1-40}$ between the no CTE/no AD, Low CTE, and Low AD groups.

\section{LATE STAGE DISEASE}

Comparing High CTE, Intermediate/High AD, and CTE + AD pathological groups, a difference in $p$-tau $231(p=0.002)$ was found overall. The High CTE group had significantly higher levels of $p$-tau 231 compared to Intermediate/High AD $(p<0.001)$ (Fig. 2). There were no significant differences in relative amounts of $A \beta_{1-42}, A \beta_{1-40,}$ ptau 181 or total tau between High CTE, Intermediate/High AD, and CTE + AD groups (Fig. 2). Although there are no significant difference between groups for ptau $_{181}$, all late stage group levels were all numerically greater than the no CTE/no AD, indicating that ptau ${ }_{181}$ was elevated in late stage disease above the no CTE/no AD group's levels as expected (Supplementary table e-1).

\section{Regression Analyses}

We performed a binary logistic regression analysis to determine the contributions of $p$-tau ${ }_{231}$ and $A \beta_{1-42}$ in predicting pathological diagnosis of CTE (low and high CTE combined, excluding CTE + AD cases) vs. no CTE/no AD. Variables 
included in the model were $p$-tau $231, A \beta_{1-42}$, age at death, and sex $(n=79)$. Both $p-\operatorname{tau}_{231}(\mathrm{OR} 1.53,95 \% \mathrm{Cl} 1.08-2.16)$ and $A \beta_{1-42}(O R 0.35,95 \%$ CI 0.17-0.74) were significant predictors of CTE status while controlling for age and sex, neither of which were significant predictors (Table 2). Secondary analyses including PMI, $\mathrm{pH}$, and presence of LBD pathology as additional variables did not substantially change the results and were not significant predictors of CTE diagnosis. Although RIN and FTLD were associated with CTE, their presence in the model did not change the associations between $\operatorname{ptau}_{231}, A \beta_{1-42}$ and CTE status.

Table 2

Binary Logistic regression comparing CTE all stages with No CTE/No $A D$ group controlling for age and sex $(n=79)$.

\begin{tabular}{|llll|}
\hline & OR & 95\% Confidence Interval & p-value \\
\hline $\mathrm{p}-\mathrm{tau}_{231}(\mathrm{U} / 10 \mu \mathrm{L})$ & 1.528 & $1.083-2.156$ & 0.016 \\
\hline $\mathrm{A} \beta_{1-42}(\mathrm{pg} / 100 \mu \mathrm{L})$ & 0.351 & $0.17-0.74$ & 0.006 \\
\hline
\end{tabular}

We also performed a binary logistic regression to determine the contributions of $p$-tau $\mathrm{u}_{231}$ and $A \beta_{1-42}$ in predicting pathological diagnosis of CTE (low and high CTE combined) vs. AD (low AD and Intermediate/High AD combined) (Table 3). Variables included in the model were $p$-tau $231, A \beta_{1-42}$, age at death, and sex $(n=113)$. Both $p$-tau $231(O R$ $1.34,95 \% \mathrm{Cl} 1.02-1.76 \mathrm{p}=0.035)$ and $\mathrm{A} \beta_{1-42}$ (OR $0.51,95 \% \mathrm{Cl} 0.28-0.91$ ) were found to distinguish between CTE and $A D$ diagnoses, controlling for age at death (OR $0.91,95 \% \mathrm{Cl} 0.84-0.97)$ and sex which was not a significant predictor. A secondary analysis adjusting for PMI $(n=104)$, demonstrated that this trend continued but with a decrease in significance most likely due to decreased power, with p-tau 231 (OR 1.24, 95\% Cl 0.90-1.70), A $\beta_{1-42}(\mathrm{OR} \mathrm{0.60,95 \%} \mathrm{Cl}$ $0.31-1.15)$, controlling for age (OR $0.88,95 \% \mathrm{Cl} 0.81-0.97)$ ), PMI (OR 1.06, 95\% Cl 1.04-1.12), and sex, which was not a significant predictor. Additional secondary analyses included the addition of RIN and pH separately as well as presence/absence of FTLD pathology and LBD pathology, none of which were significant predictors of CTE diagnosis.

Table 3

Binary Logistic regression comparing CTE all stages with $A D$ all stages controlling for age and sex $(n=113)$.

\begin{tabular}{|llll|}
\hline & OR & $95 \%$ Confidence Interval & p-value \\
\hline $\mathrm{p}-\operatorname{tau}_{231}(\mathrm{U} / 10 \mu \mathrm{L})$ & 1.340 & $1.023-1.756$ & 0.034 \\
\hline $\mathrm{A} \beta_{1-42}(\mathrm{pg} / 100 \mu \mathrm{L})$ & 0.506 & $0.283-0.907$ & 0.022 \\
\hline Age of death & 0.906 & $0.844-0.972$ & 0.006 \\
\hline
\end{tabular}

Given the surprisingly low levels of $A \beta_{1-42}$ in CTE, we performed a secondary analysis to test the hypothesis CSF betaamyloid levels reflect brain tissue $A \beta_{1-42}$ levels in $A D$, but not in CTE. Using a linear regression it was found that a model including age, sex and CSF $A \beta_{1-42}$ levels predicted a significant amount of the variance of brain $A \beta_{1-42}$ levels among the $A D$ and non $C T E$ /non $A D$ groups combined $\left(F(3,99)=3.01, p=0.034, R^{2}=0.086\right.$, adjusted $\left.R^{2}=0.057\right)$ and that CSF $A \beta_{1-42}$ trended towards predicting frontal cortex $A \beta_{1-42}(\beta=-0.18, p=0.073)$, but that age $(\beta=0.16, p=0.12)$ and sex $(\beta=0.11, p=0.29)$ did not. In a separate linear regression among the combined CTE and non-AD/non-CTE groups the overall regression model including CSF $A \beta_{1-42}$ levels, age and sex predicted a significant amount of the 
variance of brain $A \beta_{1-42}$ levels $\left(F(3,66)=2.99, p=0.037, R^{2}=0.12\right.$, adjusted $\left.R^{2}=.08\right)$, though CSF $A \beta_{1}-42$ levels $(\beta=$ $-0.15, p=0.20)$ and $\operatorname{sex}(\beta=-0.18, p=0.16)$ were not found to be a significant predictors of cortical $A \beta_{1}-42$ levels while age was found to be a significant predictor $(\beta=0.36, p=0.01)$.

\section{Receiver Operating Characteristic curve (ROC) Analyses}

To assess the diagnostic accuracy of CSF $p-t_{231}$ and $A \beta_{1-42}$ levels an ROC analysis was performed to determine if p-tau ${ }_{231}, A \beta_{1-42}$, age at death, sex were predictive of CTE vs. no CTE/no AD diagnosis. Area under the curve (AUC) was 0.89 (SEM $=0.04, p<0.001)$ (Fig. 3A). A separate ROC analysis was performed for CTE vs. AD diagnosis to determine if $p$-tau $231, A \beta_{1-42}$, age at death and sex were predictive and demonstrated that $A U C$ was $0.94(S E M=0.023, p<0.001)$ (Fig. 3B).

\section{Discussion:}

Our results indicate that in post-mortem CSF, p-tau 231 levels were significantly higher in both low and high stage CTE when comparing to low and Intermediate/High stage AD, respectively. Furthermore-and surprisingly-CSF $A \beta_{1-42}$ was found to be decreased in low stage CTE compared to the no CTE/no AD group. Overall, increased levels of $p$-tau 231 and decreased levels of $A \beta_{1-42}$ in combination were found to be significant predictors of CTE compared to nonCTE/non-AD and AD.

As CTE is defined pathologically by abnormally phosphorylated tau accumulation within neurons, astrocytes, and cell processes (7), increased p-tau 231 may be due to tau leakage from cells into the CSF, which may be present even relatively early on in the disease course when clinical symptoms are relatively mild. $\mathrm{p}$-tau 231 was significantly increased in both low and high stage CTE groups compared to AD groups, which could reflect increased neuron and axonal damage in CTE. Studies in AD have revealed that increased phosphorylated tau levels, including both $\mathrm{p}$-tau 181 and $p$-tau ${ }_{231}$, correlate with neocortical neurofibrillary burden in $\operatorname{AD}(16,17)$, though a similar relationship in CTE between elevated CSF $\mathrm{p}$-tau 231 levels and increased $\mathrm{p}$-tau accumulation in the neocortex has not been wellinvestigated. Additionally, p-tau 231 levels may inversely correlate with cognition in subjects with mild cognitive impairment $(\mathrm{MCl})$, who are at risk of progression to $A D$ dementia (18). It is not yet clear whether p-tau 231 CSF levels also inversely correlate with cognition among subjects with CTE. While t-tau may be a more general marker of neurodegeneration reflecting axonal injury in both CTE and AD, CSF p-tau 181 and p-tau 231 may be specific for the intraneuronal tau pathology seen in both CTE and AD. Our findings from the current study suggest that $p$-tau ${ }_{231} \mathrm{CSF}$ levels may be more sensitive and specific than both $\mathrm{t}$-tau and $\mathrm{p}$-tau $\mathrm{t}_{181}$ in distinguishing CTE from non-CTE and non-AD participants as well as in distinguishing CTE from AD.

The role of $A \beta$ accumulation in CTE pathophysiology is not well understood. In the current study the Low CTE group exhibited lower $A \beta_{1-42}$ levels in post-mortem CSF compared to those in the no CTE/no AD group. Decreased CSF $A \beta_{1-}$ ${ }_{42}$ in combination with elevations in tau species and relatively unchanged $A \beta_{1-40}$, are well-established markers of $A D$, and predict the conversion of mild cognitive impairment (MCI) to $A D(19-21)$. In vivo studies of former NFL players with objective memory deficits have not been found to have increased $A \beta$ deposition compared to controls as measured by florbetapir PET, indicating that cognitive decline in former players at risk of possible CTE was not related to $A D$ or $A \beta$ deposition.(22) In contrast, post-mortem evidence has shown that $A \beta$ accumulation can occur in $C T E$, however it is not a consistent pathologic feature and is absent in about half of cases, tending to form diffuse rather than neuritic plaques when present (5). However, there does appear to be an age-dependent acceleration of $A \beta$ 
deposition in CTE compared to a normal autopsy population (5). Furthermore, $\mathrm{RHI}$ associated with CTE likely damages blood vessels and is associated with the development of frontal leptomeningeal cerebral amyloid angiopathy (CAA) which is distinct from AD (23). This preferential localization of $A \beta$ associated with CAA in the leptomeningeal vessels suggests that the mechanism of decreased $A \beta_{1-42}$ seen in CTE may be related to impaired CSF clearance of $A \beta$, rather than to amyloid sequestration in plaques. We found that there was a trend towards a relationship between CSF and brain levels of $A \beta_{1-42}$ in $A D$, but that there was no relationship between CSF and brain levels in CTE. This may indicate that sequestration of $A \beta_{1-42}$ in plaques may drive CSF levels of $A \beta_{1-42}$ in $A D$, but that CSF $A \beta_{1-42}$ is determined by a different mechanism in CTE.

The relationship of the two pathologies underlying AD and CTE is not well understood and as a starting point this study has sought to investigate the levels of several CSF AD biomarkers in the CTE population with the general hypothesis that alterations in these analytes would allow improved discrimination of $A D$ and CTE. Older individuals with CTE pathology are more likely to have concurrent amyloid-beta (AB) plaques consistent with $A D(9)$ and may also develop $A \beta$ accumulation at a younger age than individuals without head injuries (5). Among 177 autopsy confirmed CTE cases in the Veteran's Affairs-Boston University-Concussion Legacy Foundation (VA-BU-CLF) CTE Brain Bank, 23 cases, nearly $13 \%$ of the total had AD in addition to CTE (24). In cases where CTE co-occurs with AD, it is unclear if individuals developed a mixed pathology neurodegenerative disorder or whether there is a synergistic relationship between the two pathophysiological processes. Among a subset of cases with concurrent $A D$ and CTE and available CSF, we did not find any group differences in CSF analyte levels. Most notably, there was no decrease in CSF A $\beta_{1-40}$ compared to non-CTE and non-AD groups. We had hypothesized that the group with CTE and AD might have decreased $A \beta_{1-40}$ levels in post-mortem CSF given our previous finding of increased deposition of $A \beta_{1-40}$ in the depths of the cortical sulci compared to gyral crests(5). The negative finding in the current study was potentially due to lack of power with only a small subset of participants with both $A D$ and CTE. An additional possibility is that decreases in CSF $A \beta$ may not correlate as closely with increased $A \beta$ plaque deposition in $C T E$, a potential departure from the wellestablished inverse correlation between CSF $A \beta$ levels and $A \beta$ plaque burden in AD. Although we did not find overall group differences in $\mathrm{p}$-tau $\mathrm{u}_{181}$ and t-tau between any of the pathologic groups, the Intermediate/Severe AD did show the expected increases in $\mathrm{p}$-tau 181 and t-tau compared to the no AD/no CTE group.

When controlling for age and sex, both $p-t_{23}$ and $A \beta_{1-42}$ were able to discriminate CTE from no CTE/no $A D$ using binary logistic regressions. $\mathrm{p}$-tau $\mathrm{u}_{231}$ and $A \beta_{1-42}$ were still significant when controlling for $\mathrm{PMI}, \mathrm{RIN}$, or $\mathrm{pH}$ individually. PMI, RIN and $\mathrm{pH}$ all generally correlate with one another. $\mathrm{p}$-tau ${ }_{231}$ and $A \beta_{1-42}$ were also able to discriminate CTE from $A D$ when controlling for age and sex, but lost significance when controlling for PMI, possibly related to decreased power.

\section{Limitations}

This study is limited in that age is a major risk factor for neurodegenerative conditions and the CTE groups were younger than the AD groups. As a result, age at death was included in the regression analysis but there is still the possibility that age could act as a potential confounder in the current study. Differences in years of play generally differ by diagnosis with CTE groups having increased years of play exposure, however this variable was missing in a significant percentage of cases and as such contact sports play is a potential confounder that could not be accounted for in our models. Furthermore, the groups did not have individuals of each gender evenly distributed and the AD group had increased numbers of women compared to the CTE groups which did not have women. This is a concern as gender differences in neuropathology have previously been reported(25). However, we have attempted to correct for this in part by running regression and sensitivity analyses among men only and have found similar results for both 
ptau 231 and $A \beta_{1-42}$ compared to analyses run with both men and women. In addition, this study was autopsy-based and thus potentially subject to selection bias as individuals whose brains are donated by family may not represent the population more broadly. However, grouping by known pathology allows for definitive associations not possible in a clinical sample where the pathology is unknown. The study was also limited in that it is post-mortem and prior studies have noted that post-mortem tau levels are increased 30 fold compared to ante-mortem(26). Both the Low and High CTE groups had significantly longer PMIs than the no CTE/no AD, Low $A D$, and Intermediate/High AD groups which raises concern that a longer PMI could lead to increased tau levels in the CTE groups. We attempted to take this into account by controlling for PMI when possible. Furthermore, given its post-mortem nature, the current study serves not to set a definitive cut-off but to warrant ante-mortem comparison of these amyloid and tau levels in CSF among patients with suspected CTE and $A D$. Of note, t-tau, $p$-tau 181 and $A \beta_{42}$ have been previously evaluated during life in former professional American football players, at increased risk of CTE, and it was found that increased cumulative head impact exposure in former players predicted t-tau levels (27).

Several promising modalities have been investigated for their use as in vivo CTE biomarkers including PET imaging of p-tau (22), peripheral blood levels of total tau and exosomal tau $(28,29)$, and CSF biomarkers $(30,31)$. CSF biomarkers are particularly promising as they closely reflect the dynamic relationship of solute clearance in the glymphatic space(32), and thus may provide a window to relatively early neuropathological changes. A study of potential CSF biomarkers has revealed increases in the chemokine CCL11 may be sensitive and specific for CTE pathology (33) and could be investigated in combination with elevations in $p$-tau ${ }_{231}$ and $A \beta_{1-42}$. Future studies should investigate other tau isoforms that have recently shown promise in $A D$ including $p-t_{217}(34)$, as well as plasma biomarkers of tau isoforms $(1,35,36)$.

\section{Conclusions:}

Overall, the current study revealed that levels of post-mortem CSF p-tau 231 and $A \beta_{1-42}$ were selectively altered in groups with CTE compared to those with $A D$, and compared to those without CTE and without AD. Both $p$-tau 231 and $A \beta_{1-42}$ were also predictive of CTE diagnosis compared to $A D$ group and compared to non-CTE/non-AD groups, indicating that $\mathrm{p}$-tau $\mathrm{ta}_{231}$ represents a potentially sensitive and specific biomarker of CTE, and that decreases in CSF $A \beta_{1-42}$ should be further investigated in vivo among possible CTE patients.

\section{List Of Abbreviations:}

Cerebrospinal fluid (CSF)

chronic traumatic encephalopathy (CTE)

Alzheimer's disease (AD)

amyloid-beta $(A \beta)$

total tau (t-tau)

phosphorylated tau ( $\mathrm{p}$-tau)

Veteran's Affairs-Boston University-Concussion Legacy Foundation Brain Bank (VA-BU-CLF)

Understanding Neurologic Injury and Traumatic Encephalopathy (UNITE)

Page $14 / 21$ 
Boston University's Alzheimer's Disease Research Center (BU ADRC)

National Alzheimer's Disease Coordinating Center (NACC)

Uniform Data Set (UDS)

Framingham Heart Study (FHS)

repetitive head impact $(\mathrm{RHI})$

Lewy body disease (LBD)

frontotemporal lobar degeneration (FTLD)

Amyotrophic Lateral Sclerosis/ Motor neuron disease (ALS/MND)

One-way analysis of variance (ANOVA)

post-mortem interval (PMI)

RNA integrity number (RIN)

Receiver Operating Characteristic curve (ROC)

Area under the curve (AUC)

mild cognitive impairment (MCl)

cerebral amyloid angiopathy (CAA)

\section{Declarations:}

Ethics: All study protocols were approved by the VA Bedford Healthcare System and Boston University Medical Center Institutional Review Boards. Written and informed consent was obtained from all patients and family members participating.

Consent for publication: not applicable

Availability of data and materials: Anonymized data not published within the article will be shared upon reasonable request.

Competing Interests: Andrew E. Budson has has been a speaker for Eli Lilly, a consultant for General Electric, Eli Lilly, and Axovant. Lee E. Goldstein has received funding from the WWE and Ivivi Health Sciences. Robert A. Stern is a member of the Mackey-White Committee of the NFL Players Association. He is a paid consultant to Biogen (Cambridge, MA, USA) and Eli Lilly (Indianapolis, IN, USA). He receives royalties for published neuropsychological tests from Psychological Assessment Resources, Inc. (Lutz, FL, USA) and is a member of the Board of Directors of KingDevick Technologies (Chicago, IL, USA). Robert C. Cantu is a paid consultant to the NFL Head Neck and Spine Committee, NOCSAE, Concussion Legacy Foundation, receives royalties from book publications, and compensation from expert legal opinion. He is a member of the Mackey-White Committee of the NFL Players Association. Ann C. McKee is a member of the Mackey-White Committee of the NFL Players Association. All other authors have no competing interests or disclosures to report. 
Funding: This work was supported by the United States (U.S.) Department of Veterans Affairs, Veterans Health Administration, Clinical Sciences Research and Development Merit Awards (I01-CX001038) (CX001698-01A2); Career Development Award-2 (\# IK2 CX002065) Alzheimer's Association (NIRG-305779, NIRG-362697); National Institute of Aging (RF1AG054156, R56AG057768, RF1AG057768, K23AG046377, U19AG068753, AG08122, AG054076); National Institute of Neurological Disorders and Stroke (U54NS115266, U01NS086659, K23NS102399); National Institute of Aging Boston University AD Center (P30AG13846; supplement 0572063345-5; pilot award 50204517 ); National Heart, Lung and Blood Institute (75N92019D00031 and HHSN2682015000011); Department of Defense Peer Reviewed Alzheimer's Research Program (PRARP \#13267017); and the Concussion Legacy Foundation. This work was also supported by unrestricted gifts from the Andlinger Foundation and WWE.

Author Contributions:

KWT played a major role in drafting manuscript writing, revisions, and data analysis and interpretation.

AG helped with initial manuscript drafting and data entry and analysis.

VEA helped with data generation and provided critical manuscript review for content.

WX helped with brain and CSF ELISA data generation and analysis, critical manuscript review for content.

JDC provided assistance with data interpretation, critical manuscript review for content.

RN provided assistance with brain and CSF data generation and analyses.

GM helped with brain and CSF ELISA data generation and analysis.

SD helped with brain and CSF ELISA data generation.

YT provided assistance with statistical plan, data generation and critical manuscript review for content.

$\mathrm{BH}$ helped with data generation, analyses and critical manuscript review for content.

AEB provided assistance with data analysis and critical manuscript review for content.

BD provided assistance with data analysis and critical manuscript review for content.

NK provided assistance with data analysis and critical manuscript review for content.

RCC provided assistance with data analysis and critical manuscript review for content.

LEG provided assistance with data analysis and critical manuscript review for content.

DIK provided assistance with data analysis and critical manuscript review for content.

RAS provided assistance with data analysis and critical manuscript review for content.

MLA provided assistance with data analysis and critical manuscript review for content.

JM provided assistance with statistical plan, data analysis and critical manuscript review for content.

ACM provided assistance with data analysis and critical manuscript review for content. 
TDS led all aspects of study including concept and design, data analysis and interpretation, and critical manuscript writing and editing.

Acknowledgements: We gratefully acknowledge the use of resources and facilities at the Edith Nourse Rogers Memorial Veterans Hospital (Bedford, MA) as well as all the individuals whose participation and contributions made this work possible.

\section{References:}

1. Ashton NJ, Leuzy A, Karikari TK, Mattsson-Carlgren N, Dodich A, Boccardi M, et al. The validation status of blood biomarkers of amyloid and phospho-tau assessed with the 5-phase development framework for AD biomarkers. Eur J Nucl Med Mol Imaging. 2021.

2. Zetterberg $\mathrm{H}$, Blennow K. Moving fluid biomarkers for Alzheimer's disease from research tools to routine clinical diagnostics. Mol Neurodegener. 2021;16(1):10.

3. McGrowder DA, Miller F, Vaz K, Nwokocha C, Wilson-Clarke C, Anderson-Cross M, et al. Cerebrospinal Fluid Biomarkers of Alzheimer's Disease: Current Evidence and Future Perspectives. Brain Sci. 2021;11(2).

4. Ehrenberg AJ, Khatun A, Coomans E, Betts MJ, Capraro F, Thijssen EH, et al. Relevance of biomarkers across different neurodegenerative diseases. Alzheimers Res Ther. 2020;12(1):56.

5. Stein TD, Montenigro PH, Alvarez VE, Xia W, Crary JF, Tripodis Y, et al. Beta-amyloid deposition in chronic traumatic encephalopathy. Acta Neuropathol. 2015;130(1):21-34.

6. Mez J, Solomon TM, Daneshvar DH, Murphy L, Kiernan PT, Montenigro PH, et al. Assessing clinicopathological correlation in chronic traumatic encephalopathy: rationale and methods for the UNITE study. Alzheimers Res Ther. 2015;7(1):62.

7. McKee AC, Cairns NJ, Dickson DW, Folkerth RD, Keene CD, Litvan I, et al. The first NINDS/NIBIB consensus meeting to define neuropathological criteria for the diagnosis of chronic traumatic encephalopathy. Acta Neuropathol. 2016;131(1):75-86.

8. Adams JW, Alvarez VE, Mez J, Huber BR, Tripodis Y, Xia W, et al. Lewy Body Pathology and Chronic Traumatic Encephalopathy Associated With Contact Sports. J Neuropathol Exp Neurol. 2018;77(9):757-68.

9. McKee AC, Stern RA, Nowinski CJ, Stein TD, Alvarez VE, Daneshvar DH, et al. The spectrum of disease in chronic traumatic encephalopathy. Brain. 2013;136(Pt 1):43-64.

10. Alosco ML, Cherry JD, Huber BR, Tripodis Y, Baucom Z, Kowall NW, et al. Characterizing tau deposition in chronic traumatic encephalopathy (CTE): utility of the McKee CTE staging scheme. Acta Neuropathol. 2020;140(4):495512.

11. Boyle PA, Yu L, Wilson RS, Leurgans SE, Schneider JA, Bennett DA. Person-specific contribution of neuropathologies to cognitive loss in old age. Ann Neurol. 2018;83(1):74-83.

12. Karanth S, Nelson PT, Katsumata Y, Kryscio RJ, Schmitt FA, Fardo DW, et al. Prevalence and Clinical Phenotype of Quadruple Misfolded Proteins in Older Adults. JAMA Neurol. 2020.

13. McKeith IG. Consensus guidelines for the clinical and pathologic diagnosis of dementia with Lewy bodies (DLB): report of the Consortium on DLB International Workshop. J Alzheimers Dis. 2006;9(3 Suppl):417-23.

14. Mackenzie IR, Neumann M, Bigio EH, Cairns NJ, Alafuzoff I, Kril J, et al. Nomenclature and nosology for neuropathologic subtypes of frontotemporal lobar degeneration: an update. Acta Neuropathol. 2010;119(1):1-4.

15. Cairns NJ, Bigio EH, Mackenzie IR, Neumann M, Lee VM, Hatanpaa KJ, et al. Neuropathologic diagnostic and nosologic criteria for frontotemporal lobar degeneration: consensus of the Consortium for Frontotemporal Lobar 
Degeneration. Acta Neuropathol. 2007;114(1):5-22.

16. Buerger K, Ewers M, Pirttila T, Zinkowski R, Alafuzoff I, Teipel SJ, et al. CSF phosphorylated tau protein correlates with neocortical neurofibrillary pathology in Alzheimer's disease. Brain. 2006;129(Pt 11):3035-41.

17. Tapiola T, Alafuzoff I, Herukka SK, Parkkinen L, Hartikainen P, Soininen H, et al. Cerebrospinal fluid \{beta\}-amyloid 42 and tau proteins as biomarkers of Alzheimer-type pathologic changes in the brain. Arch Neurol. 2009;66(3):382-9.

18. Buerger K, Teipel SJ, Zinkowski R, Blennow K, Arai H, Engel R, et al. CSF tau protein phosphorylated at threonine 231 correlates with cognitive decline in MCl subjects. Neurology. 2002;59(4):627-9.

19. Hampel H, Burger K, Teipel SJ, Bokde AL, Zetterberg H, Blennow K. Core candidate neurochemical and imaging biomarkers of Alzheimer's disease. Alzheimers Dement. 2008;4(1):38-48.

20. Hansson O, Zetterberg H, Buchhave P, Londos E, Blennow K, Minthon L. Association between CSF biomarkers and incipient Alzheimer's disease in patients with mild cognitive impairment: a follow-up study. Lancet Neurol. 2006;5(3):228-34.

21. Mattsson N, Zetterberg H, Hansson O, Andreasen N, Parnetti L, Jonsson M, et al. CSF biomarkers and incipient Alzheimer disease in patients with mild cognitive impairment. JAMA. 2009;302(4):385-93.

22. Stern RA, Adler CH, Chen K, Navitsky M, Luo J, Dodick DW, et al. Tau Positron-Emission Tomography in Former National Football League Players. N Engl J Med. 2019;380(18):1716-25.

23. Standring OJ, Friedberg J, Tripodis Y, Chua AS, Cherry JD, Alvarez VE, et al. Contact sport participation and chronic traumatic encephalopathy are associated with altered severity and distribution of cerebral amyloid angiopathy. Acta Neuropathol. 2019;138(3):401-13.

24. McKee AC, Stein TD, Kiernan PT, Alvarez VE. The neuropathology of chronic traumatic encephalopathy. Brain Pathol. 2015;25(3):350-64.

25. Mielke MM, Vemuri P, Rocca WA. Clinical epidemiology of Alzheimer's disease: assessing sex and gender differences. Clin Epidemiol. 2014;6:37-48.

26. Morihara T, Kudo T, Ikura Y, Kashiwagi Y, Miyamae Y, Nakamura Y, et al. Increased tau protein level in postmortem cerebrospinal fluid. Psychiatry Clin Neurosci. 1998;52(1):107-10.

27. Alosco ML, Tripodis Y, Fritts NG, Heslegrave A, Baugh CM, Conneely S, et al. Cerebrospinal fluid tau, Abeta, and sTREM2 in Former National Football League Players: Modeling the relationship between repetitive head impacts, microglial activation, and neurodegeneration. Alzheimers Dement. 2018;14(9):1159-70.

28. Stern RA, Tripodis Y, Baugh CM, Fritts NG, Martin BM, Chaisson C, et al. Preliminary Study of Plasma Exosomal Tau as a Potential Biomarker for Chronic Traumatic Encephalopathy. J Alzheimers Dis. 2016;51(4):1099-109.

29. Shahim P, Tegner Y, Wilson DH, Randall J, Skillback T, Pazooki D, et al. Blood biomarkers for brain injury in concussed professional ice hockey players. JAMA Neurol. 2014;71(6):684-92.

30. Washnik NJ, Anjum J, Lundgren K, Phillips S. A Review of the Role of Auditory Evoked Potentials in Mild Traumatic Brain Injury Assessment. Trends Hear. 2019;23:2331216519840094.

31. Muraoka S, Jedrychowski MP, Tatebe H, DeLeo AM, Ikezu S, Tokuda T, et al. Proteomic Profiling of Extracellular Vesicles Isolated From Cerebrospinal Fluid of Former National Football League Players at Risk for Chronic Traumatic Encephalopathy. Front Neurosci. 2019;13:1059.

32. Nedergaard M. Neuroscience. Garbage truck of the brain. Science. 2013;340(6140):1529-30.

33. Cherry JD, Stein TD, Tripodis Y, Alvarez VE, Huber BR, Au R, et al. CCL11 is increased in the CNS in chronic traumatic encephalopathy but not in Alzheimer's disease. PLoS One. 2017;12(9):e0185541. 
34. Janelidze S, Stomrud E, Smith R, Palmqvist S, Mattsson N, Airey DC, et al. Cerebrospinal fluid p-tau217 performs better than p-tau181 as a biomarker of Alzheimer's disease. Nat Commun. 2020;11(1):1683.

35. Simren J, Leuzy A, Karikari TK, Hye A, Benedet AL, Lantero-Rodriguez J, et al. The diagnostic and prognostic capabilities of plasma biomarkers in Alzheimer's disease. Alzheimers Dement. 2021.

36. Ashton NJ, Pascoal TA, Karikari TK, Benedet AL, Lantero-Rodriguez J, Brinkmalm G, et al. Plasma p-tau231: a new biomarker for incipient Alzheimer's disease pathology. Acta Neuropathol. 2021.

\section{Figures}

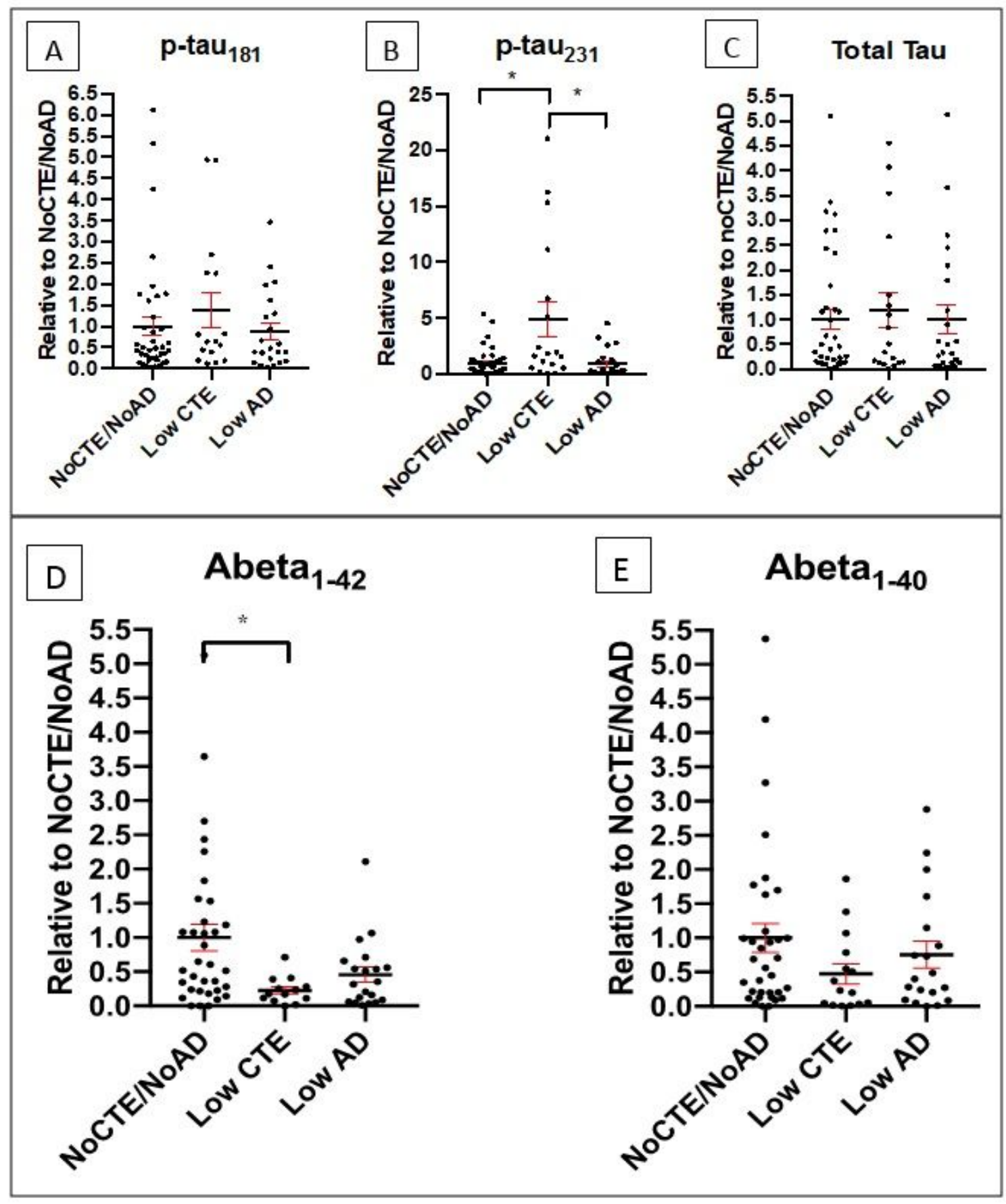

Figure 1 
Fold change of A. p-tau181, B. p-tau231, C. total tau, D. Aß1-42 and E. Aß1-40 for no CTE/no AD, Low CTE, and Low AD groups. Scatter plots show individual values, mean value and SEM as bars, ${ }^{*} \mathrm{p}<0.016$ corrected for multiple comparisons; ANCOVA correcting for age.
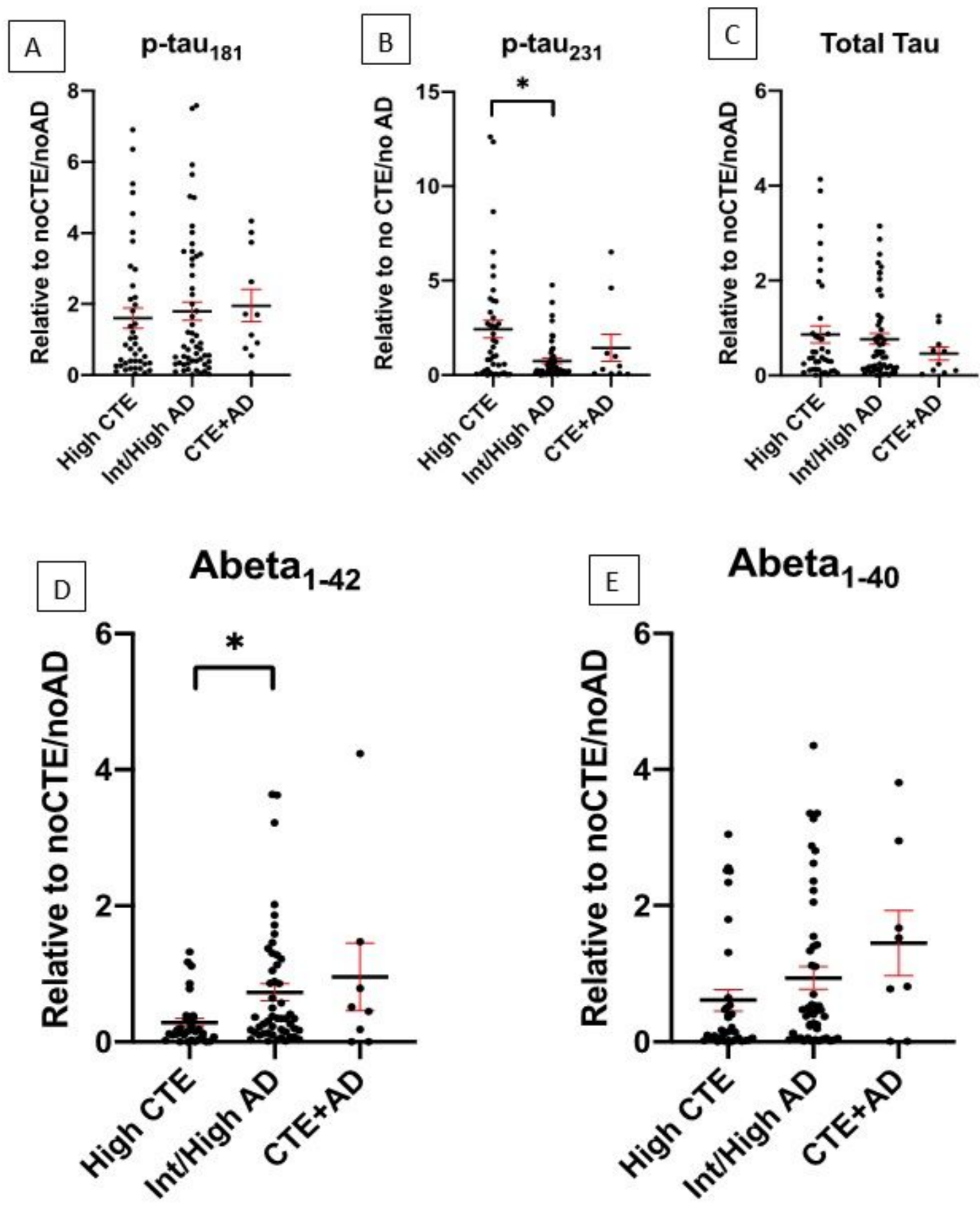

Figure 2

Fold change of A. p-tau181, B. p-tau231, C. total tau, D. Aß1-42 and E. AB1-40 for High CTE, Intermediate/High AD, and $C T E+A D$ groups. Scatter plots show individual values, mean value and $S E M,{ }^{*} p<0.016$ corrected for multiple comparisons; ANCOVA correcting for age. 


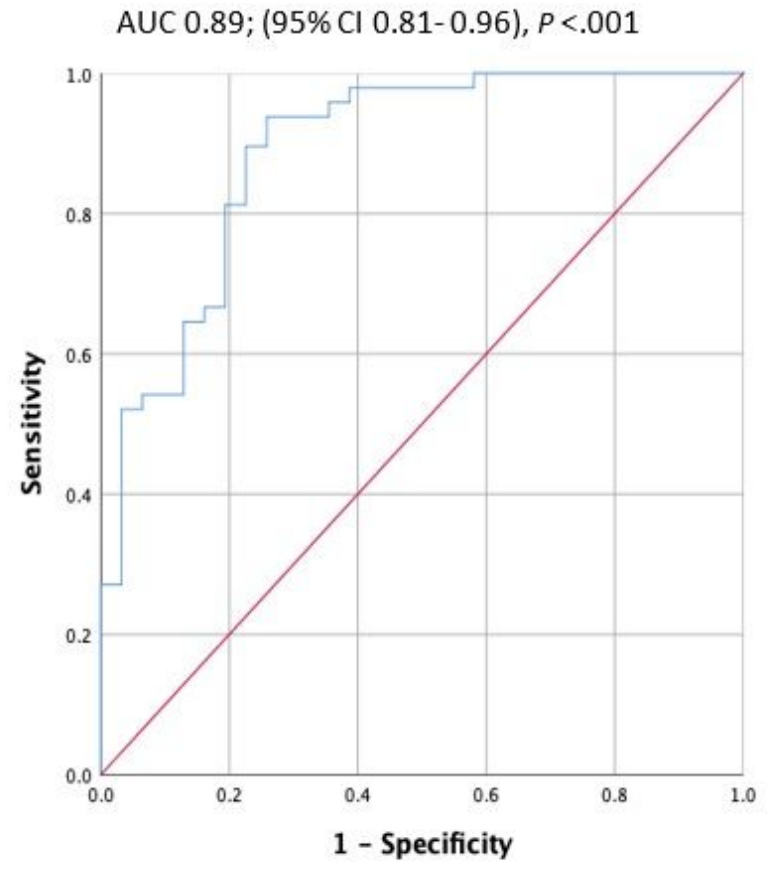

A
AUC 0.94; (95\% Cl 0.89- 0.98), $P<.001$

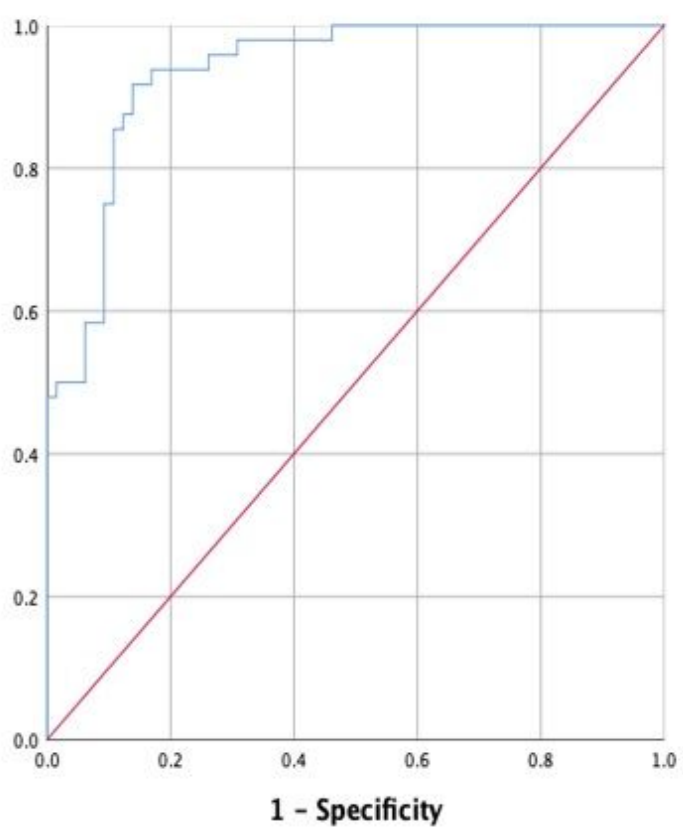

B

\section{Figure 3}

Diagnostic accuracy of $(A)$-tau231, A 1 1-42, age at death, sex for CTE vs. no CTE/no AD diagnosis $(n=79)$ and $(B) p$ tau231, $A \beta 1-42$, age at death and sex were predictive of CTE vs. AD $(n=113)$. AUC = Area under the receiver operating characteristic curve; $\mathrm{Cl}=$ confidence interval.

\section{Supplementary Files}

This is a list of supplementary files associated with this preprint. Click to download.

- Tablee1e23.16.21424PM.docx

- SupplementalResults3.17.211054AM.docx 